\title{
Nursing Activities Score: an updated guideline for its application in the Intensive Care Unit
}

\author{
Nursing activities score: manual atualizado para aplicação em Unidade de Terapia Intensiva \\ Nursing activities score: manual actualizado para su \\ aplicación en la Unidade de Cuidados Críticos \\ Katia Grillo Padilha1, Siv Stafseth², Diana Solms², Marga Hoogendoom ${ }^{3}$, Francisco Javier Carmona Monge ${ }^{4}$, Om Hashem \\ Gomaa $^{5}$, Konstantinus Giakoumidakis ${ }^{6}$, Margarita Giannakopoulou ${ }^{6}$, Maria Cecília Gallani ${ }^{7}$, Edyta Cudak ${ }^{8}$, Lilia de Souza \\ Nogueira ${ }^{1}$, Cristiane Santoro ${ }^{1}$, Regina Cardoso de Sousa ${ }^{1}$, Ricardo Luis Barbosa ${ }^{9}$, Dinis dos Reis Miranda ${ }^{10}$
}

${ }^{1}$ Universidade de São Paulo, Escola de Enfermagem, Departamento de Enfermagem Médico-Cirúrgica, São Paulo, SP, Brazil.

${ }^{2}$ Oslo University Hospital, Division of Emergencies and Critical Care, Oslo, Norway.

${ }^{3}$ Isala klinieken Research Department Anesthesiologie \& Intensive Care, Zwolle, the Netherlands.

${ }^{4}$ Hospital Universitario Fundación Alcorcón, Madrid, España.

${ }^{5}$ Sohag University, Faculty of Nursing, Sohag, Egypt.

${ }^{6}$ University of Athens, Faculty of Nursing, Athens, Greece.

${ }^{7}$ Université Laval, Faculté des Sciences Infirmières, Quebec, Canada.

${ }^{8}$ Poznan University of Medical Sciences, Faculty of Health Sciences, Poznan, Poland

${ }^{9}$ Universidade Federal de Uberlândia, Instituto de Geografia, MG, Brazil.

${ }^{10}$ University of Groningen, Former Researcher, Groningen, the Netherlands.

\section{ABSTRACT}

Objective: To describe nursing workload in Intensive Care Units (ICU) in different countries according to the scores obtained with Nursing Activities Score (NAS) and to verify the agreement among countries on the NAS guideline interpretation. Method: This cross-sectional study considered 1-day measure of NAS (November 2012) obtained from 758 patients in 19 ICUs of seven countries (Norway, the Netherlands, Spain, Poland, Egypt, Greece and Brazil). The Delphi technique was used in expertise meetings and consensus. Results: The NAS score was $72.8 \%$ in average, ranging from $44.5 \%$ (Spain) to $101.8 \%$ (Norway). The mean NAS score from Poland, Greece and Egypt was 83.0\%, $64.6 \%$ and $57.1 \%$, respectively. The NAS score was similar in Brazil (54.0\%) and in the Netherlands (51.0\%). There were doubts in the understanding of five out 23 items of the NAS (21.7\%) which were discussed until researchers' consensus. Conclusion: NAS score were different in the seven countries. Future studies must verify if the fine standardization of the guideline can have a impact on differences in the NAS results.

\section{DESCRIPTORS}

Workload; Nursing, Team; Nursing care; Intensive Care Units; Handbooks.
Correspondence Addressed to:

Katia Grillo Padilha

Av. Dr. Enéas de Carvalho Aguiar,

419 - Cerqueira César

CEP 05403-000 - São Paulo, SP, Brazil

kgpadilh@usp.br
Received: 04/10/2015

Approved: 07/14/2015 


\section{INTRODUCTION}

There is evidence that nursing workload is associated with the quality of patient care and the health of workers ${ }^{(1-5)}$. Since the 1970s, the gold standard for nurse staffing levels in intensive care and subsequently critical care units has been one nurse for each patient ${ }^{(6)}$. However, critical care has changed substantially since that time and in recent years this standard has been challenged both in number of nurses and skill-mix.

In this context, tools for assessing nursing workload were developed $^{(7-9)}$. Among these tools there are several models of the Therapeutic Interventions Scoring System (TISS) ${ }^{(10-13)}$, one of which is the Nursing Activities Score (NAS) which was launched in $2003^{(14)}$. The NAS has been validated by means of a study of 99 ICUs in 15 countries $^{(14)}$. It is a modified version of TISS-28 with an additional five items, namely monitoring and titration, hygiene procedures, mobilization and positioning of the patient, support and care of relatives/patients, and administrative and management tasks plus medical interventions. The NAS weightings measure the time consumed by nurse activities at the patient level and represent the percentage of nursing time (one in a 24-hour period) dedicated to the performance of the activities included in the instrument. The sum of the weights of the individual items scored reflects the amount of time spent by nursing staff in an ICU on performing activities during a particular day. Results indicated that the NAS accounts for $81 \%$ of the nursing time, whereas TISS- 28 accounts for only $43 \%{ }^{(14)}$. Thus, NAS has been translated into many languages and is in use in 12 countries including Norway and Finland in the global north, and Brazil in the south ${ }^{(15-22)}$.

More than ten years of use and experiences have been published. However, some problems were observed in its application by intensive care nurses around the world. The main difficulties were related to the lack of clear operational explanations about certain items. Although NAS has its own specific instructions for use ${ }^{(15)}$ the definitions do not solve all doubts arising from this "old" manual application and new methods, when used in interventions and treatments. This issue motivated the network to perform a multi-center study and explore the problem to provide an updated guideline.

The main objectives were to: describe NAS scores in different countries and verify the agreement among countries on the NAS guideline interpretation.

\section{METHOD}

This cross-sectional multicenter study was developed in 19 ICUs in seven countries: Norway, the Netherlands,
Spain, Poland, Egypt, Greece and Brazil, considering 1-day measure of NAS (November 2012).

The eligibility criteria for ICU inclusion were adult patients, general or specialized type and current use of NAS.

The sample consisted of patients consecutively admitted to the ICUs, aged 16 years and more, undergoing medical or surgical treatment with a length of stay in the ICU for at least 24 hours.

Demographic and clinical variables included age, length of stay (LOS), severity of illness (Simplified Acute Physiologic Score II-SAPS II), discharge of the ICU (survival or non survival) and nursing workload (total NAS score and item by item score).

The project was approved by the hospital and research ethics committees of each country. Medical records were used to obtain demographic data, LOS, SAPS II and NAS indexes. All the indexes were collected in the first 24 hours in the ICU.

After the data collection and analysis, a second phase of the study, the Delphi technique involved a meeting of the group of researchers in Sao Paulo, Brazil, in earlyNovember 2014. The objective of this meeting was to discuss different interpretations of NAS items and to establish a consensus for the drawing up of an updated version of the NAS manual. As a reference for discussion, the instrument's original manual was used $^{(15)}$. In light of the results gathered during data collection in the participating countries, each item from the instrument was discussed until a consensus was reached among all participants with regard to interpretation of the item. In cases of divergences, DRM (coordinator of NAS development and validation) was consulted to facilitate reaching a consensus.

Following on from this procedure, a second, electronic meeting was held among the researchers at the end of November 2014 to adjust the results. Subsequently a final meeting in-person took place in Valencia, Spain in January 2015 when the NAS manual was updated for application in clinical practice in the ICU.

Data were entered in Google Docs (Egypt, Poland, the Netherlands and Greece) and Excel (Sapin, Braxil and Norway) and afterwards submitted to descriptive analysis in Brazil, using version 19.0 of the SPSS software.

\section{RESULTS}

A sample of 758 patients from 19 ICUs in seven countries, of whom $61.1 \%$ were male, $53.7 \%$ were submitted to clinical treatment and 8,2\% died in the Unit.

Demographic and clinical data are shown in Table 1.

Table 1 - Number of patients, age, LOS, SAPSII, NAS score and death in the different countries.

\begin{tabular}{lcccccc}
\hline ICU & Patients n (\%) & Age mean (SD) & LOS mean (SD) & SAPSII mean (SD) & NAS mean (SD) & Death n (\%) \\
\hline EGY & $39(5.1)$ & $40.7(19.1)$ & $6.5(1.0)$ & $37.3(20.8)$ & $57.1(10.0)$ & $13(33.3)$ \\
GRE & $66(8.7)$ & $65.0(11.6)$ & $2.0(0.3)$ & $28.9(13.9)$ & $64.6(4.7)$ & $16(24.2)$ \\
NET & $109(14.4)$ & $65.0(13.3)$ & $6.7(8.3)$ & $32.7(15.8)$ & $51.0(11.5)$ & $9(8.3)$ \\
POL & $23(3.0)$ & $61.8(13.9)$ & $8.3(15.9)$ & $65.0(12.9)$ & $83.0(14.7)$ & $2(9.5)$ \\
SPA & $54(7.1)$ & $65.9(13.2)$ & $5.9(6.1)$ & $37.8(15.0)$ & $44.5(13.0)$ & $5(10.2)$ \\
BRA & $182(24.0)$ & $67.6(17.5)$ & $3.3(5.8)$ & $30.9(21.9)$ & $54.0(6.1)$ & $10(5.5)$ \\
NOR & $285(37.6)$ & $62.9(16.9)$ & $3.9(3.9)$ & $33.8(11.9)$ & $101.8(31.3)$ & $7(2.5)$ \\
\hline Total & $758(100,00)$ & $63.5(16.9)$ & $4.4(6.2)$ & $33.94(17.3)$ & $72.8(31.1)$ & $62(8.2)$ \\
\hline
\end{tabular}

$\mathrm{SD}=$ Standard Deviation. 
Table 1 shows that a higher percentage of patients from the ICUs of Norway, Brazil and the Netherlands (37.6\%, $24.0 \%$ and $14.4 \%$, respectively), corresponding to $76.0 \%$ of the sample. These were followed by the ICUs in Greece, Spain, Egypt and Poland, that together admitted $24.0 \%$ of the patients.

The mean age of all patients was 63.5 years and ranged from 61.8 to 67.6 years in the units of all countries, except in Egypt where the patients were younger (mean age: 40.7 years).

The mean LOS ranged from 2.0 to 3.9 days in the ICUs from 3 of the 7 countries (Greece, Brazil and Norway). A higher mean LOS (about 6 days or longer) was observed in the ICUs in Spain, Egypt, the Netherlands and Poland.

Regarding the severity of illness, the mean SAPS II of the sample was 33.9 points and ranged from 30.9 to 37.8 points in most countries (71.4\%). The lowest severity was observed in Greece (28.9 points) and the highest in Poland (65.0 points).

The highest mortality rates were observed at the Egyptian and Greek ICUs (33.3\% and 24.2\%), and lowest rates at the Brazilian and Norwegian ICUs (2.5\% and 5.5\%). The mortality rates at the ICU of other countries were: $10.2 \%$ in Spain, 9.5\% in Poland and 8.3\% in the Netherlands.

The mean nursing workload of the total sample was high, at $72.8 \%$. The highest mean NAS score was obtained for the Norway ICU (101.8\%), followed by Poland (83.0\%) and Greece (64.6\%). With the exception of the Spanish ICU which scored lowest (44.5\%), scores ranged from $51.0 \%$ to $57.1 \%$ in the Netherlands, Brazil and Egypt.

Concerning the understanding of the 23 NAS items, there was a consensus among the researches on the interpretation and scores for the majority of items (18/78.3\%): items $1 \mathrm{a}, \mathrm{b}$ and $\mathrm{c}$ (monitoring and tritation); item 2 (laboratory); item 3 (medication: vasoactive drugs excluded), items $4 \mathrm{a}, \mathrm{b}$ and $\mathrm{c}$ (hygiene procedures); item 5 (care of drains); items 6a, b and c (mobilization and positioning); items $7 \mathrm{a}$ and $\mathrm{b}$ (support and care of relatives and patient); item 9 (respiratory support); item 10 (care of artificial airways); item 11 (treatment for improving lung function); item12 (vasoactive medication); item 13 (intravenous replacement of large fluid losses); item 16 (hemofiltration techniques); item 17 (quantitative urine output measurement); item 18 (measurement of intracranial pressure); item 20 (intravenous hyperalimentation); item 21 (enteral feeding) and item 23 (specific interventions outside the intensive care unit; surgery or diagnostic procedures).

On the other hand, there was a disagreement concerning the interpretation of 5 items: item $8 \mathrm{c}$ (performing administrative and managerial tasks requiring full dedication for about 4 hours or more...); item 14 (left atrium monitoring); item 15 (cardiopulmonary resuscitation after arrest); item 19 (treatment of complicated metabolic acidosis/alkalosis) and item 22 (specific intervention(s) in the intensive care unit).

The main questions concerning these items were: must we consider item $8 \mathrm{c}$ if there are nursing students under supervision of the bedside nurse?; is it possible to consider in item 14 the use of procedures such as intra-aortic balloon pumping, extracorporeal life support (ECLS), ventricular assistance devices?; must we consider patients who suffered cardiac arrest (item 15) within the last 24 hours even when outside the ICU or only in the ICU?; must we consider in item 19 only acute treatments such as bolus of sodium bicarbonate or can we also consider when the patient is in hemofiltration, when the solution is buffered and in 24 hours the minimal amount is given, as laid out in the original manual?; can we include the procedures mentioned in item 22 , if it takes more time or do we need to include an exhaustive list of possible procedures to include in this item as examples?

After orientation from the NAS author and discussion among the researchers in the meetings, consensus was reached in Valencia, in January 2015, and the NAS manual was concluded as follows.

Chart 1 - Instruction manual for implementation of NAS

\begin{tabular}{|c|c|}
\hline BASIC ACTIVITIES & Score \\
\hline \multicolumn{2}{|l|}{ 1. MONITORING AND TITRATION } \\
\hline $\begin{array}{l}\text { 1a. Hourly vital signs, regular registration and calculation of fluid balance } \\
\text { Patients who require NORMAL monitoring, according to the ICU routine, of vital signs, application of assessment scales (pain, RASS, } \\
\text { Glasgow), water balance control (including nasogastric and nasoenteral tubes) and who do not need frequent alterations in treatment, } \\
\text { therapy or monitoring intensification. Assisted oral feeding. }\end{array}$ & 4.5 \\
\hline $\begin{array}{l}\text { 1b. Present at bedside and continuous observation or active for } 2 \text { hours or more in any shift, for reasons of safety, severity or therapy, } \\
\text { such as: non-invasive mechanical ventilation, weaning procedures, restlessness, mental disorientation, prone position, donation } \\
\text { procedures, preparation and administration of fluids and/or medication, assisting specific procedures. } \\
\text { Patients who require intensified monitoring (MORE THAN NORMAL) due to alterations in the clinical condition, hemodynamic instability, } \\
\text { oliguria, bleeding, dyspnea, fever, alteration in the level of consciousness, measurements in the assessment scales higher than the ICU standard, } \\
\text { measurement of central venous pressure, invasive arterial pressure, intra-abdominal pressure, use of sedatives or long-term use of insulin, ventilator } \\
\text { support, non-invasive mechanical ventilation or alteration of the ventilator parameters, preparation of fluids and emergency medication. Patient } \\
\text { is stable after the therapeutic behavior adopted. Immediate post-operative care after cardiac surgery or major surgery, where the patient remains } \\
\text { stable. Invasive procedures with intercurrences. Extubation without intercurrences. Assisted oral feeding that demands more time than normal. }\end{array}$ & 12.1 \\
\hline $\begin{array}{l}\text { 1c. Present at bedside and active for } 4 \text { hours or more in any shift for reasons of safety, severity or therapy, such as those examples above (1b). } \\
\text { Critical patients who require MUCH MORE THAN NORMAL monitoring, in at least one shift in } 24 \text { hours, without stabilization after the } \\
\text { therapeutic interventions adopted, requires continuous nursing presence. Alterations described in the "MORE THAN NORMAL" category, } \\
\text { however with a greater frequency and the need for interventions. Hemodialysis with intercurrence, requiring nursing intervention (when } \\
\text { hemodialysis is performed by ICU staff). Unstable patients in immediate postoperative care after cardiac surgery or major surgery. }\end{array}$ & 19.6 \\
\hline $\begin{array}{l}\text { 2. LABORATORY: Biochemical and microbiological investigations. } \\
\text { Patients submitted to any biochemical or microbiological exam, regardless of the quantity, performed at bedside by a nursing professional, } \\
\text { including capillary glucose. E.g.: HGT, glycosuria, tracing cultures, blood gas analysis, among others. This item should not be scored if the } \\
\text { laboratory collector or physician performs the collection. }\end{array}$ & 4.3 \\
\hline
\end{tabular}


3. MEDICATION: Vasoactive drugs excluded.

Patients who received any type of medication, regardless of the route and dose. Vasoactive drugs will be scored in a specific item (item 12).

\section{HYGIENE PROCEDURES}

Performing hygiene procedures such as: dressing of wounds and intravascular catheters, changing linen, washing patient, incontinence, vomiting, burns, leaking wounds, complex surgical dressing with irrigation, special procedures (e.g. barrier nursing, cross-infection related, room cleaning following infections, staff hygiene) and especially obese patients, etc.

\section{4a. NORMAL}

Patients who were submitted, in NORMAL frequency (ICU routine), to one of the hygiene procedures mentioned above in at least one shift in 24 hours. Also including dressings closed in vascular catheter once a day.

4b. The performance of hygiene procedures took more than 2 hours in any shift.

Patients who were submitted, in MORE THAN NORMAL frequency, to one of the hygiene procedures mentioned above in at least one shift in 24 hours. Vascular catheter dressing twice a day; medium dressing for pressure ulcer, dressing a surgical incision twice a day, medium dressing (with suture dehiscence); changing linen twice in $24 \mathrm{~h}$; washing of unstable patients by three professionals; body hygiene twice per shift. Fecal incontinence three times a day. Patients in isolation.

4c The performance of hygiene procedures took more than 4 hours in any shift.

Patients who were submitted, in MUCH MORE THAN NORMAL frequency, to one of the hygiene procedures mentioned above in at least one shift in 24 hours. Extensive, complex, open cavity dressing or $\geq$ three times a day.

5. CARE OF DRAINS - All (except gastric tube).

Patients with any type of drain or tube with the aim of draining. Including long-term catheter, external ventricular drain (EVD), thorax drain, among others. EXCLUDING gastric tubes (nasogastric, nasoenteral, gastrostomies and others), which should be considered in item 1 or 21

6. MOBILIZATION AND POSITIONING

Including procedures such as: turning the patient; mobilization of the patient; moving from bed to chair; team lifting (e.g. immobile patient, traction, prone position).

6a. Performing procedure(s) up to 3 times per 24 hours.

Patients who require mobilization and positioning up to three times in 24 hours.

6b. Performing procedures(s) more frequently than 3 times per 24 hours, or with 2 nurses - any frequency.

Patients who require mobilization and positioning, as described in item 6 , which have been performed more than three times in 24 hours or by two members of the nursing staff in at least one shift in 24 hours.

6c. Performing procedure with three or more nurses - any frequency.

Complex mobilization and positioning as per the procedure described in item 6 , which have been performed by three or more members of the nursing staff, in any frequency, in at least one of the shifts in 24 hours.

\section{SUPPORT AND CARE OF RELATIVES AND PATIENT}

Including procedures such as telephone calls, interviews, counseling. Often, the support and care of either relatives or patient allow staff to continue with other nursing activities (e.g.: communication with patients during hygiene procedures, communication with relatives whilst present at bedside and observing patient).

7a. Support and care of either relatives or patient requiring full dedication for about one hour in any shift such as: to explain clinical condition, dealing with pain and distress, difficult family circumstances.

This item receives a score when guidance or instructions are given to patients and/or their families, providing emotional support with full dedication of a nurse from the staff, with NORMAL duration, according to the routine established in the unit, in at least one shift in 24 hours.

7b. Support and care of either relatives or patient requiring full dedication for 3 hours or more such as: to explain clinical condition, dealing with pain and distress, difficult family circumstances.

This item receives a score when guidance or instructions are given to patients and/or their families, providing emotional support with full dedication of a nurse from the staff, with MORE THAN NORMAL duration, according to the routine established in the unit, in at least one shift in 24 hours.

\section{ADMINISTRATIVE AND MANAGERIAL TASKS}

8a. Performing routine tasks such as: processing of clinical data, ordering examinations, professional exchange of information (e.g.: ward rounds). Including records performed as nursing process and/or shift change, multidisciplinary rounds or administrative and managerial tasks related to patients, with NORMAL duration.

8b. Performing administrative and managerial tasks requiring full dedication for about 2 hours in any shift such as: research activities, protocols in use, admission and discharge procedures.

Including records performed as part of nursing process and/or shift change, multidisciplinary rounds or administrative and managerial tasks related to patients, with MORE THAN NORMAL duration. Admission of patients in immediate postoperative period, unstable patients who require more extensive records. Need for providing materials and equipment. Assembly of the hemodialysis machine, application of protocols such as ECLS, transplantation, others. When the nurse needs help from a colleague to perform his/her activities. E.g.: the nurse continues assisting a patient and a colleague takes over the administrative tasks.

8c. Performing administrative and managerial tasks requiring full dedication for about 4 hours or more of the time in any shift such as: death and organ donation procedures, co-ordination with other disciplines.

Including any administrative and managerial task related to the patient, with MUCH MORE THAN NORMAL duration, according to the routine established in the unit. Critical, unstable patients who require intense records. Detailed shift change records, multidisciplinary rounds, organization of special materials and equipment for patient care, surgical procedures at bedside, protocols such as transplantation, ECLS, ventricular assist devices, teaching and supervising education/training.

\section{VENTILATORY SUPPORT}

9. Respiratory support: Any form of mechanical ventilation/assisted ventilation with or without positive end-expiratory pressure, with or without muscle relaxants; spontaneous breathing with positive end-expiratory pressure (e.g. CPAP or BiPAP), with or without endotracheal tube; supplementary oxygen by any method.

Patients making use of any respiratory support, from nasal catheter to mechanical ventilation.

10. Care of artificial airways. Endotracheal tube or tracheostomy cannula.

Patients making use of orotracheal or nasotracheal tube or tracheostomy. 
11.Treatment for improving lung function. Lung physiotherapy, incentive spirometry, inhalation therapy, intratracheal suctioning.

\section{CARDIOVASCULAR SUPPORT}

12. Vasoactive medication, irrespective of type or dose.

Patients who have received any vasoactive medication, regardless of the type and dose and who need intensive monitoring in their

endovenous use: Sodium Nitroprusside, Vasopressin, Prostaglandin, Norepinephrine, Epinephrine, Dopamine, Dopexamine, Dobutamine,

Isoproterenol, Phenylephrine, Nitroglycerin, Clonidin hydrochloride. Metoprolol and Propranolol (beta blockers) should be scored.

13. Intravenous replacement of large fluid losses. Fluid administration $>3 \mathrm{l} / \mathrm{m}^{2} /$ day, irrespective of type of fluid administered.

Patients who have received fluid replacement greater than 4.5 liters of solution per day, irrespective of the type of fluid administered.

14. Left atrium monitoring. Pulmonary artery catheter with or without cardiac output measurement.

Patients making use of pulmonary artery catheter (Swan-Ganz catheter). Including the use of cardiac pacemaker, intra-aortic balloon pumping, cardiac output monitoring, extracorporeal life support (ECLS), ventricular assist devices.

15. Cardiopulmonary resuscitation after arrest; in the past 24 hours (single precordial thump not included).

Patients who suffered a heart problems and were submitted to cardiopulmonary resuscitation, independently of the environment where the cardiac arrest took place. This item should be scored only once in 24 hours.

\section{RENAL SUPPORT}

16. Hemofiltration techniques. Dialysis techniques.

Patients who have received any type of intermittent or continuous dialytic procedure.

17. Quantitative urine output measurement (e.g.: by indwelling urinary catheter).

Patients who require diuresis control, in milliliters, with or without any type of urinary device.

\section{NEUROLOGICAL SUPPORT}

\section{Measurement of intracranial pressure.}

Patients submitted to intracranial pressure monitoring, jugular bulb catheter or microdialysis. Do consider this item if the patient has external ventricular drainage and assessment of ICP.

\section{METABOLIC SUPPORT}

19. Treatment of complicated metabolic acidosis/alkalosis.

Patients who made use of specific medication to adjust metabolic acidosis or alkalosis, such as administration of sodium bicarbonate in continuous or bolus infusion. Respiratory acidosis and alkalosis should not be scored in this item, neither should ventilator

correction. The item considers those conditions requiring the permanent presence of a nurse for monitoring severe physiological

deregulation and for titrating (fine-tuning) the therapy in acute conditions. During hemofiltration, if correction is necessary,

additional score is indicated.

20. Intravenous hyperalimentation.

Patients who receive central or peripheral venous infusion of parenteral nutrition.

21. Enteral feeding. Through gastric tube or other gastrointestinal route (e.g., jejunostomy).

Patients who receive enteral feeding through tubes, by any route of the gastrointestinal tract. Measurement of aspiration/retention included.

\section{SPECIFIC INTERVENTIONS}

22. Specific intervention(s) in the intensive care unit. Endotracheal intubation, insertion of pacemaker, cardioversion, endoscopies, emergency surgery in the past 24 hours, gastric lavage. Routine interventions without direct consequences for the clinical condition of the patient, such as: X-rays, echography, electrocardiogram, dressing, or insertion of venous or arterial catheters, are not included.

Patients submitted to a diagnostic or therapeutic intervention listed above in the ICU. Specific procedures performed in the unit and which require active intervention of the staff can be considered in this item, including the insertion of venous or arterial catheters and spinal puncture. Procedures performed by the nurse, such as passing a relief or indwelling urinary catheter, a nasoenteral or gastric tube, a peripherally inserted central catheter (PICC), installation of intra-abdominal pressure, among others, that might be particular complex and require more nursing time for their execution can also be considered.

\section{Specific interventions outside the intensive care unit. Surgery or diagnostic procedures.}

Patients who require diagnostic or therapeutic interventions performed outside the ICU. E.g.: tomography, radionuclide imaging, magnetic resonance, hemodynamics (take or pick up a patient), surgical procedures (take or pick up a patient), patient transfer to any hospitalization unit or discharge, and sending the body to the morgue.

Observations: The sub-items of item 1,4,6,7 and 8 are mutually exclusive as well as NORMAL, MORE THAN NORMAL and MUCH MORE THAN NORMAL In the calculation of the total NAS, the greatest score obtained in the items 1, 4, 6, 7 and 8 in the 24 hour period will be considered.

\section{DISCUSSION}

The workload of nurses in ICUs in countries that participated in the research, an average of $72.8 \%$, was consistent with that found in other studies that applied the NAS in different $\mathrm{ICUs}^{(16,18,24)}$.

However, the NAS measures showed a great degree of variation between countries with a minimum of $44.5 \%$ (Spain) and a maximum of $101.8 \%$ (Norway), as well as average values of $83.0 \%$ (Poland), $64.59 \%$ (Greece) and 57.1\%, 54.0\% and 51.0\% (Egypt, Brazil and the Netherlands, respectively). Although similar values have been re- ported by other researchers ${ }^{(20-21,25-26)}$, it is possible to attribute these differences to the type of ICU, as well as to the characteristics of the patients.

Among the total sample of patients, the average age was 63.5, with a SAPS II score of 33.9 points, a length of stay in the ICU of 4.4 days and a mortality rate of $8.2 \%$. These results, however, different varied among countries. These differences can explain at least partially the variation in the workload of nurses and need to be explored further.

The present study sought to go beyond just describing the workload of nurses in ICUs in the participating countries, and, 
through the application of NAS by experienced nurses, aimed to verify the consensus in the understanding of each item of the instrument and guideline, with the objective of providing an updated and standardized manual for use in clinical practice.

The analysis and discussion of the results of each item, separately, indicated that the researchers are in agreement in their understanding of the majority (78.26\%) of items in the NAS. However, there were doubts relating to $5(21.7 \%)$ items, whether because of a lack of clarity in the original manual or with regard to new processes and interventions that did not exist when the instrument was first established in 2003.

It is possible to conclude that the understanding shown by the researchers in relation to the majority of items about which no doubts arose occurred on account of previous communication between the researchers, mainly during the initial phase of implementation of the NAS in the ICUs. Nevertheless, independently of their understanding of these items, all of them were presented during discussions and consensus was reached by participants.

With regard to the five items for which there are doubts, these relate to item 8 (include or not the activities for accompanying students in the ICU as sub-item ' $c$ '); item 14 (consider or not new procedures); item 15 (score or not care for cardio-respiratory arrest in the previous 24 hours, when occurring outside of the ICU); item 19 (score or not bicarbonate repositioning and, if necessary, during the process of hemofiltration) and item 22 (draw up or not a list of the different procedures to be scored, exceptionally when more time was asked for to carry them out).

After discussion with the NAS author and further discussion between the researchers, consensus was reached by all regarding these doubts, and conclusions were included in the manual.

The updating of and proposal for a set of guidelines for completing the NAS in ICUs, after more than a decade of applying the instrument, arose due to a need for a better understanding and uniformity of its use among researchers and intensive care nurses. The correct application of the tool will make it possible to measure the real working demands of nurses in the ICU. Assessment of NAS do contribute towards a more effective investment of human and material resources at the unit, and as a result, improvements in the quality of care, greater job satisfaction and a reduction in costs.

\section{CONCLUSION}

The nursing workload observed in the ICUs of the seven countries that participated in the study showed a great degree of variation of average NAS, from a minimum of $44.5 \%$ to a maximum of $101.8 \%$. Discussion on these results provided important input to the updated and standardized NAS instruction manual for use in clinical practice. The appropriate application of NAS will support the taking up of real measures in response to the working demands of nurses and will allow for improved investment in human and material resources at the ICU.

\section{RESUMO}

Objetivo: Descrever a carga de trabalho de enfermagem em Unidades de Terapia Intensiva (UTI) de diferentes países, segundo o Nursing Activities Score (NAS), e padronizar o manual do NAS para uso nessas Unidades. Método: Estudo transversal realizado em 19 UTI de sete países (Noruega, Holanda, Espanha, Polônia, Egito, Grécia e Brasil) com um total de 758 pacientes adultos, em novembro de 2012. Resultados: A média do NAS total da amostra foi 72.81\%, com variação entre 44.46\% (Espanha) e101.81\% (Noruega). Nas UTI da Polônia, Grécia e Egito, as médias foram de 83.00\%, 64.59\% e 57.11\%, respectivamente. As médias NAS no Brasil (53.98\%) e na Holanda (50,96\%) foram similares. Dos 23 itens da escala, houve dúvidas no entendimento de 5(21.74\%), que foram solucionados por consenso entre os pesquisadores. Conclusão: $\mathrm{O}$ estudo mostrou diferentes cargas de trabalho de enfermagem nas UTI estudadas. Um manual padronizado do NAS para uso nessas unidades contribuirá para sanar dúvidas em futuras aplicações.

\section{DESCRITORES}

Carga de Trabalho; Equipe de Enfermagem; Cuidados de Enfermagem; Unidades de Terapia Intensiva; Manuais.

\section{RESUMEN}

Objetivo: Describir la carga de trabajo de enfermería en Unidades de Cuidados Intensivos (UCI) de diferentes países según el Nursing Activities Score (NAS) y establecer una guía estandarizada para su utilización en UCI. Método: estudio observacional en 19 UCIs de siete países (Noruega, Países Bajos, España, Polonia, Egipto, Grecia y Brasil) incluyendo 758 pacientes adultos en Noviembre de 2012. Resultados: La puntuación media total en la escala NAS fue de $72.81 \%$ com valores entre $44.46 \%$ (España) y $101.8 \%$ (Noruega). Las medias NAS en Polonia, Grecia y Egipto fue de 83.0\%, 64.59\% y 57.11\% respectivamente. El NAS medio fue similar en Brasil (53.98\%) y los Países Bajos (50.96\%). De los 23 ítems de la escala hubo problemas en la interpretación de 5 de ellos (21.74\%). Este problema se resolvió mediante el consenso entre los investigadores. Conclusión: El presente estudio demuestra variación en la carga de trabajo en UCI de diferentes países. La guía estandarizada de puntuación del NAS puede servir como una herramienta para resolver dudas en futuras aplicaciones.

\section{DESCRIPTORES}

Carga de Trabajo; Grupo de Enfermería; Atención de Enfermería; Unidades de Cuidados Intensivos; Manuales. 


\section{REFERENCES}

1. Penoyer DA. Nurse staffing and patient outcomes in critical care: a concise review. Crit Care Med. 2010;38(7):1521-8.

2. Kane RL, Shamliyan TA, Mueller C, Duval S, Wilt TJ. The association of registered nurse staffing levels and patient outcomes: systematic review and meta-analysis. Med Care. 2007;45(12):1195-204.

3. Aiken L, Sloane DM, Bruyneel L, Griffiths P, Sermeus W, Busse R, et al. Staffing and education of nurses and hospital mortality in EuropeAuthors' reply. Lancet. 2014;384(9946):851-2.

4. Numata Y, Schulzer M, Van der Wal R, Globerman J, Semeniuk P, Balka E, et al. Nurse staffing levels and hospital mortality in critical care settings: literature review and meta-analysis. J Adv Nurs. 2006;55(4):435-48.

5. Aiken L, Sermeus W, Heede KV, Sloane DM, Busse R, McKee M, et al. Patient safety, satisfaction and quality of hospital care: cross sectional surveys of nurses and patients in 12 countries in Europe and the United States. BMJ. 2012;20(344):1-14.

6. Bray K, Wren I, Baldwin A, Ledger US, Gibson V, Goodman S, et al. Standards for nurse staffing in critical care units. Nurs Crit Care. 2010;15(3):109-11.

7. Italian Multicenter Group of ICU Research (GIRTI). Time Oriented Score System (TOSS): a method for direct and qualitative assessment of nursing workload for ICU patients. Intensive Care Med. 1991;17(6):340-5.

8. Fagerstrom L, Rauhala A. Benchmarking in nursing care by the RAFAELA patient classification system - a possibility for nurse managers. J Nurs Manag. 2007; 15(7):683-92.

9. Intensive Care National Audit and Research Centre. System of Patient Related Activities (SOPRA). London: ICNARC; 1999.

10. Cullen DJ, Civetta JM, Briggs BA, Ferrara LC. Therapeutic Intervention Scoring System: a method for quantitative comparison of patient care. Crit Care Med. 1974;2(2):57-60.

11. Keene AR, Cullen DJ. Therapeutic Intervention Scoring System: update. Crit Care Med. 1983;11(1):1-3.

12. Miranda DR, De Rijk A, Schaufeli W. Simplified therapeutic intervention scoring system: the TISS-28 items - result from a multicenter study. Crit Care Med.1996;24(1):64-73.

13. Miranda DR, Moreno R, lapichino G. Nine equivalents of nursing manpower score (NEMS). Intensive Care Med. 1997;23(7):760-5.

14. Miranda DR, Nap R, De Rijk A, Schaufeli W, lapichino G. Nursing activities score (NAS). Crit Care Med. 2003;31(2):374-82.

15. Padilha KG, Sousa RMC, Queijo AF, Mendes AM, Miranda DR. Nursing Activities Score in the intensive care unit: analysis of the related factors. Intensive Crit Care. 2008;24(3):197-204 .

16. Stafseth SK, Solms D, Bredal IS. The characterisation of workloads and nursing staff allocation in intensive care units: a descriptive study using the Nursing Activities Score for the first time in Norway. Intensive Crit Care Nurs. 2011;27(5):290-4

17. Ducci AJ, Zanei SSV, Whitaker IY. Nursing workload to verify nurse/patient ratio in a cardiology ICU. Rev Esc Enferm USP. 2008;42(4):673-80.

18. Giakoumidakis K, Baltopoulos GI, Charitos C, Patelarou E, Fotos NV, Brokalaki-Pananoudaki H. Risk factors for increased in-hospital mortality: a cohort study among cardiac surgery patients. Eur J Cardiovasc Nurs. 2012;11(1):23-33.

19. Adell AB, Abizanda Campos R, Yvars Bou M, Quintana Bellmunt J, Gascó Garcia C, Soriano Canuto M, et al. Care work load in critical patients. Comparative study NEMS versus NAS. Enferm Intensiva. 2006;17(1):67-77.

20. Adell AB, Abizanda CR, Cubedo RM, Quintana BJ, Sanahuja RE . Nursing Activity Score (NAS). Our experience with a nursing load calculation system based on times. Enferm Intensiva. 2005;16(4):164-73.

21. Deberg D, Myny D, Herzeele VI, Maele GV, Miranda DR, Colardyn F. Measuring the nursing workload per shift in the ICU. Intensive care Med. 2012; 38(9):1438-44.

22. Queijo AF, Martins RS, Andolhe R, Oliveira EM, Barbosa RL, Padilha KG. Nursing workload in neurological intensive care units: crosssectional study. Intensive Crit Care Nurs. 2013;29(2):112-6.

23. Nursing Activities Score: instructions for use. Crit Care Med [Internet]. 2004 [cited 2015 Feb 22]. Available from: http://download.Iww. com/wolterskluwer_vitalstream_com/PermaLink/CCM/A/00003246-920030200-00001.pdf

24. Nogueira LS, Domingues CA, Poggetti RS, Sousa RMC. Nursing workload in intensive care unit trauma patients: analysis of associated factors. Plos One. 2014; 9(11):e112125.

25. Nogueira LS, Koike KM, Sardinha DS, Padilha KG, Sousa RMC. Nursing workload in public and private intensive care units. Rev BrasTer Intensiva. 2013;25(5):225-32.

26. Carmona-Monge FJ, Jara-Perez A, Quirós-Herranz C, Rollán-Rodríguez G, Cerrillo-González I, García-Gómez S, et al. Assessment of nursing workload in three groups of patients in a Spanish ICU using the Nursing Activities Score Scale. Rev Esc Enferm USP. 2013;47(2):335-40. 